\title{
Introducing the Nepal Journal of Epidemiology: A Pioneer in its Domain
}

\author{
Sathian B ${ }^{1}$
}

${ }^{1}$ Assistant Professor, Department of Community Medicine, Manipal College of Medical Sciences, Pokhara, Nepal

\section{Editorial}

\section{Corresponding Author:}

Dr. Brijesh Sathian, Assistant Professor, Department of Community Medicine, Manipal College of Medical Sciences, Pokhara, Nepal.

Email:drsathian@gmail.com

The Nepal Journal of Epidemiology is the first journal of its kind in Nepal. Our main objective in pioneering this journal is to attempt to provide a common platform for all researchers, particularly those doing epidemiological studies on prevailing public health problems in the community. All who use, receive, or pay for health-care interventions depend on guidance from reliable research findings and will want reassurance that medical research is credible. Essentially, Nepal's research output is still small. More collaboration and partnerships between countries in different regions of Asia and other parts of the globe as well, must be fostered. Lack of investment in research should also be addressed by nations which are capable of making investments. Research in Nepal can and should flourish over in the coming decades.

We aspire to make this journal one that follows a standard criteria of scientific article writing combined with sound technical proficiency. Several initiatives have been set up that will improve the quality of reporting and we have given prime importance to the transparency of research in general, and randomised trials in particular, by emphasising the importance of protocols. We offer to review protocols to improve trial quality and lessen publication bias. We consider submissions of randomised trials only if registered and accompanied by a protocol, which is sent with the manuscript to peer reviewers.

A brief review through almost any recently published medical journal will show that statistical methods play an increasingly important role in modern medical research. Many research papers quote at least one ' $p$-value' to communicate their results while some present the results and the statistical analysis of medical data in relatively sophisticated and complex sets ${ }^{1-8}$.

After extensive study of the available literature and from personal experience in this domain, I would like to venture a few recommendations for the improvement of various aspects in medical research and its application. I believe that this brief discussion will be of great value to all professionals involved in medical research and its application.

"Medicine is a science of uncertainty and an art of probability", mused William Osler. Medical journals are a confluence of medicine, science and journalism-and are expected to have the values of all three. Medical journals differ fundamentally from scientific journals in that the former is read mainly by practising doctors and not by scientists. Medical journals will continue to be the main vehicle of scientific information for years to come, particularly where access to computer and internet facilities are relatively limited. Nowadays the output-and rewardsof research are based almost entirely on published papers in scientific journals. Scientists in low-income and middleincome settings want an opportunity to analyze data for their populations according to their own concerns. They want to be in the frontlines of national and global communications about their country's experiences. Evidence-based medicine provides several ways to quantify and communicate uncertainty, but does so from a probabilistic rather than a human perspective. We can divide Evidence based medicine/clinical epidemiology into two major methodological themes: 'statistical' and 'implementation'. The use and analysis of large trials, metaanalyses, systematic reviews, evidence hierarchies, costeffectiveness analyses and the number needed to treat come under the 'statistical' methodology while the improved access to evidence through literature searching, library and critical appraisal tools, guideline development, risk framing, etc. would be 'implementation'. Researchers welcome clinical uncertainty as a source of knowledge gaps, whose answers will be meaningful to clinicians and patients. Clinical epidemiology bridges clinical practice and public 
health.

Policies notwithstanding, training in research ethics, standards and responsible conduct is often minimal or absent in academia, despite suggestions to detect and eliminate research misconduct. The quality of a medical journal depends on the inclusion of three groups of people: the authors, reviewers and editors. Deciding who should be listed as an author is not simple and quite often the decision is made on the basis of supremacy.

Scientific reading will enhance the quality of scientific writing. Critical reading and thinking will provide a relevant, interesting, feasible, ethical and novel research hypothesis. The author or researcher planning on a research study and publication should search in Medline, PubMed and other search engines for relevant reviews and literature of similar studies in world and national scenario. These studies must be examined for strengths and weaknesses, and the researcher must apply required modifications for new knowledge. It will help the author tremendously in the writing of the introduction, discussion and conclusion of the manuscript.

Before starting data collection, the researcher should decide upon the study design, target population, sample size and sampling method, inclusion exclusion criteria, study period, study variable, outcome measures and units of measurement, definition of all the terms and variables and their classification. After careful consideration, the methodology of data collection and the method of data analysis, including the computer packages and statistical methods, should be chosen. The instruments or questionnaire used to measure the variables should be described correctly and if others have developed them, referenced properly. A researcher should be well aware of the concepts of different types of data and variables, two types of errors (type I and type II errors), calculation of sample size, significance level, confidence interval, testing of hypothesis and power of the test ${ }^{1-11}$. Once these criteria are followed, the authors should allot paragraphs for each one of them in the material and methods part of the manuscript, thereby increasing the quality of the study. This meticulous planning and execution will be useful to new researchers in this area.

The vital part of the publishing process is the editorial management. The editors commence action with the receipt of the manuscript by directing the various steps of evaluation, correction and re-submission, until an editorial decision is taken to accept the paper as is, accept it after modification or reject it if it is unacceptable. They then carry out the necessary text and layout editing. Appropriate concern is given to the statistical and ethical aspects as well as to the overall uniformity of the terminology, nomenclature and style throughout the volume as a whole. Experts review plays a pivotal role on maintaining the quality of a medical journal. A reviewer's duty is to comment on important aspects of the paper and to formulate recommendations relating to the acceptability of the paper.
Findings of good research deserve to be presented well, and good presentation is as much a part of the study as is the painstaking collection and analysis of the data. Critical appraisal of 150 articles published in a reputed medical journal in Nepal revealed that in more than $70 \%$ articles experienced biostatisticians were not involved or had no substantial contribution (not co authored); in more than $65 \%$ of the studies, the sample size calculation was wrong and $80 \%$ of articles had inadequate statistical details and wrong statistical tests.

Critical reviewers of biomedical literature have consistently found that nearly half of the published articles (including scientific articles, published even in the best journals) used statistical methods contained unacceptable errors ${ }^{1-11}$.

The term "statistics" here in this context, has a wider meaning and includes the methodology of research, study design, or epidemiological methodology, etc ${ }^{1-8}$. The major applications of biostatistics started in the middle of the 17th century in the analysis of vital statistics. After the early developments in vital statistics, the field of genetics was the next area that benefited most from the new statistical ideas emerging in the works of Charles Darwin (1809-1882), Francis Galton (1822-1910), Karl Pearson (1857-1936), and Ronald A. Fisher (1890-1962). Now, the fields of application and areas of concern of biostatistics include evidence based medicine, bioassays, public health, health service research, nutrition, environmental health, demography, epidemiology, surveys of human populations, community diagnosis, bio-mathematical modelling, clinical trials, brain imaging, genomics and proteomics. Computer-based statistical software programs are not yet capable enough to decide the appropriate method although they sometimes generate a warning message in case of inadequate data. Hence, the user of the software has to decide the correct statistical analysis method ${ }^{9}$.

The real solution to poor statistical reporting will come when authors learn more about the statistical methods in research design and when statisticians are able to convey the importance of the methods used in the study to authors, editors, and readers; when researchers begin to involve statisticians from the beginning of research, not at its end; when manuscript editors begin to understand and apply statistical reporting and editing guidelines; when the journals are able to screen the articles containing statistical analyses more carefully; and when readers learn more about how to interpret statistics and begin to expect and demand, adequate statistical reporting ${ }^{7}$. A researcher should never hesitate to ask for professional assistance from a biostatistician to plan the study or experiment.

Despite valuable research going on in developing countries and the large number of existing scientific journals, this information does not reach international visibility. Not only are such journals obscure, but they also perpetuate a vicious circle of inadequacy that may directly be damaging the local science and research culture. Journals should prevent this by constructing an editorial board including qualified editors from developed and developing countries 
in the editorial board.

I recommend biostatisticians to join as editors and reviewers in order to help formulate journal policy, audit the quality of statistics in published papers, help produce statistical guidelines or checklists for authors, educate editors, provide explanatory statistical comments on published papers and write expository articles about statistical matters in journals.

\section{Conflict of Interests}

The author has no conflict of interest arising from the study.

\section{References}

1. Glantz SA. Biostatistics: how to detect, correct and prevent errors in the medical literature. Circulation 1980; 61(1): 1-7.

2. Gore SM, Jones G, Thompson SG. The Lancet's statistical review process: areas for improvement by authors. Lancet 1992;340(8811): 100-2.

3. Hemminki E. Quality of reports of clinical trials submitted by the drug industry to the Finnish and Swedish control authorities. Eur J Clin Pharmacol 1981;19(3):157-65.

4. Herbst AL, Ulfelder $H$, Poskanzer DC. Adenocarcinoma of the vagina. Association of maternal stilbestrol therapy with tumor appearance in young women. N Engl J Med 1971; 284(15): 878-81.

5. Hill OW Jr. Rethinking the "significance" of the rejected null hypothesis. Am Psychol 1990; 45: 667-8.

6. The coronary primary prevention trial: design and implementation: the Lipid Research Clinics Program. J Chronic Dis 1979; 32(9-10): 609-31.

7. Matthews DE, Farewell VT. Using and understanding medical statistics. 3rd ed. Basel, Switzerland: S. Karger AG, 1966.

8. Schor S, Karten I. Statistical evaluation of medical journal manuscripts. JAMA 1966; 195(13): 1123-8.

9. Indrayan A. Statistical fallacies in orthopedic research. Indian J Orthop 2007;41(1):37-46.

10. Yim KH, Nahm FS, Han KA, Park SY. Analysis of statistical methods and errors in the articles published in the korean journal of pain. Korean J Pain 2010; 23(1): 35-41.

11. Jin $Z$, Yu D, Zhang L, Meng $H$, Lu J, et al. A retrospective survey of research design and statistical analyses in selected Chinese medical journals in 1998 and 2008. 2010; 5(5):10822. 